\title{
On the Automobile Tire Pressure Intelligent Detection and Control System on the Basis of the Single-Chip Microcomputer
}

\author{
Zhenhua Wang ${ }^{1, a}$, Meng Jin $^{1, b}$ and Zhentao Liü, c \\ ${ }^{1}$ College of electrical and information, Dalian Jiaotong University, China

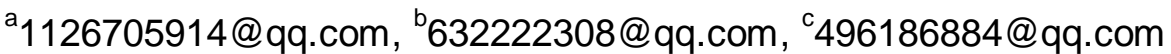

\begin{abstract}
Keywords: Single-chip microcomputer; Tire pressure monitoring; Control system; MPX4105; System
\end{abstract} simulation.

\begin{abstract}
The paper designs an automobile tire pressure intelligent detection and control system on the basis of the single-chip microcomputer so as to realize the real-time monitoring of the automobile tire pressure and control the automobile tire pressure through the corresponding executing agency. When the tire pressure is higher than the given maximum value or lower than the given minimum value, the system will raise the audible and visual alarm. Moreover, the system sets the temperature monitoring device to raise alarm when the temperature is above the set value and lower the temperature through the cooling device specific to the certain situations that the tire may be damaged due to the excess temperature. The system displays the corresponding information with the 1602 liquid crystal display (LCD) with good human-computer interaction interface. The simulation operation in the proteus environment shows that the system has simple operation, high accuracy and good real-time performance and it has reaches the expected design purpose.
\end{abstract}

\section{Introduction}

Thanks to the rapid popularization of the automobile, the pursuit of people is higher and higher for the driving safety and comfort. The onboard digital barometer can guarantee the safe driving of people and effectively reduce the probability of traffic accident due to tire burst. The service provider needs a tire pressure detection and control system attractive in price and quality. It makes full use of the function of MPX4105 chips to meet the needs of the collection, control and data processing of the automobile tire gauge and improve the system stability and anti-interference ability. Meanwhile, it simplifies the circuit design with easy adjustment and more balanced indicators so as to greatly reduce the cost because a lot of work can be realized through the single-chip microcomputer software. In addition, the tire pressure detection and control system has good function expansibility with the advantages of high accuracy, good stability and easily extendable function, etc as a kind of powerful platform. It provides conditions for the subsequent technology upgrading of the instruments and electronic product design so as to further meet the requirements of the market [1].

It designs and realizes an automobile tire pressure detection and control system on the basis of the single-chip microcomputer mainly specific to the design of the tire gauge of the automobile tire on account of the above introduction. The tire gauge of the automobile tire gains the analog voltage value corresponding to the automobile tire pressure through the air pressure sensor and process by inputting to the single-chip microcomputer through the V/F transform so as to display the corresponding pressure values in real time. The parameters shall be carefully understood when the digital pressure barometer is designed in case of the damage of the tire gauge due to improper use. The tire gauge of the automobile tire adopts the high-performance absolute pressure sensors with its screen display of the high accurate automobile tire pressure in order to realize the real-time monitoring of the tire pressure [2]. When the automobile tire pressure is in the operation of the abnormal state, it will inform the driver through the alarm. Meanwhile, the system also has the function of temperature monitoring to prevent the occurrence of tire burst and achieve the purpose of safe driving. 


\section{The Overall Design of the System}

The Overall Function Design of the System. The measured pressure converts to the analog voltage output through the pressure sensor when measuring so the output signal cannot be directly processed by a single-chip microcomputer. Therefore, the analog voltage outputted from the air pressure sensor needs to be converted to the digital pulse through the A/D conversion module. The frequency of the digital pulse presents the linear variation with the input voltage. The pulse signal is received though the single-chip microcomputer to gain the obtained pulse counts in the unit interval. The corresponding actual pressure value is calculated according to the linear relation between the voltage and frequency [3]. In the end, it shows to the users through LCD display circuit. The overall block diagram of the system is shown in Fig. 1:

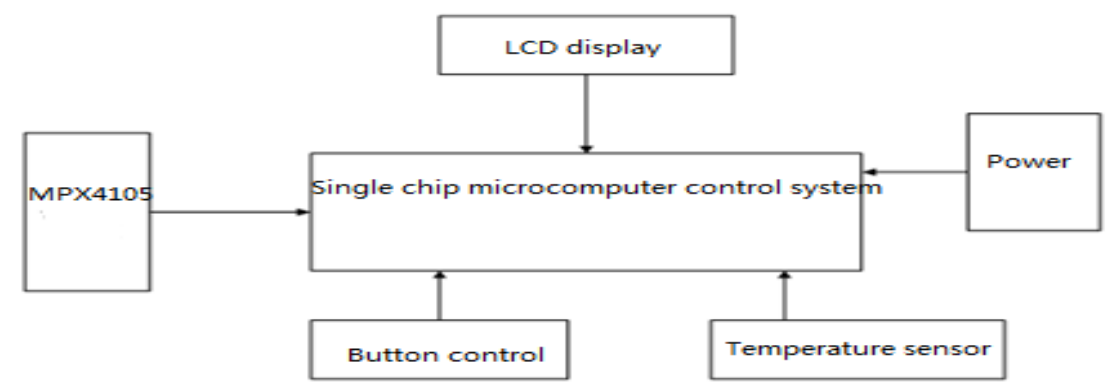

Figure 1. The overall block diagram of the system

It is designed through the setting of each port of the single-chip microcomputer and the selection of the working mode of the timer and the serial port. Moreover, the timer and serial port is initialized to realize the setting of the communication and contact between the single-chip microcomputer and the chips of each functional module. In the main program module, the key is to initialize the single-chip microcomputer and distribute the variables in the programs of the address space, etc. The most critical one is to connect each functional module of the subroutines.

The Overall Hardware Design of the System. The system adopts the master control of the integrated single-chip microcomputer to convert the pressure signal to the AT89C52 single-chip microcomputer with A/D conversion through the pressure sensor. What's more, it conducts A/D conversion and other data processing aided by the corresponding simulation discrete components and sends the processing results to display in the displaying part.Moreover, the system also adds the temperature detection and alarm system in addition to the main functions above. It can detect the tire temperature with power-off protection to further strengthen the practicability of the system and ensure the driving safety to a large extent[4]. The overall hardware diagram is shown in Fig. 2.

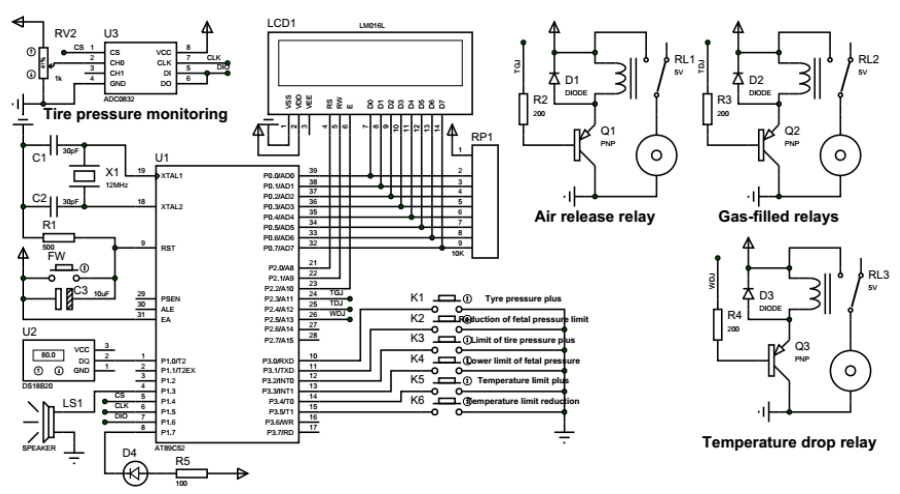

Figure 2. Hardware diagram of the system

The Overall Design of the System Software. It is designed through the setting of each port of the single-chip microcomputer and the selection of the working mode of the timer and the serial port. 
Moreover, the timer and serial port is initialized to realize the setting of the communication and contact between the single-chip microcomputer and the chips of each functional module. In the main program module, the key is to initialize the single-chip microcomputer and distribute the variables in the programs of the address space, etc. The most critical one is to connect each functional module of the subroutines.

First of all, timer 0 and counter 1 are set. It returns when the counter is in a state of 0 . It comes into the timer 0 service subroutine when the counter is in a state of 1 . When ET0 $=0$, it starts counting and then determines whether the count is 10 . When it is $10, \mathrm{ET} 0=1$ and TR $1=1$ are set. When it is not 10 , TR $1=0$ and counter restoration are set. Then the pressure value is calculated and the display function is called to back ET0 $=1$ and TR $1=1$ again.

This design uses $\mathrm{C}$ language as programming tools. $\mathrm{C}$ language is a compiling structured programming language with simple grammatical structure and powerful processing capabilities. It has the advantages of fast operation, high efficient compiling, good portability and readability, etc to realize the direct operation of the system hardware. It can greatly shorten the development cycle and obviously increase the readability of the software to compile the software of the target system with $\mathrm{C}$ language. What's more, it is convenient for the improvement and expansion so as to develop the large-scale and high-powered application system[5]. In addition, $\mathrm{C}$ language provides the storage types of auto, static and flash, etc to automatically distribute the space for the variables reasonably specific to the program storage space, data storage space and EEPROM space of the single-chip microcomputer. What's more, C language provides complex data types to greatly enhance the program processing power and flexibility. $\mathrm{C}$ compiler can automatically realize the site protection and recovery of the interrupt service routines, and provide commonly used standard function library, for the use of the user. Moreover, $\mathrm{C}$ complier can automatically generate the initial code of some hardware and can realize the development of some complex systems through the real-time operation of the transplant or provided by $\mathrm{C}$ complier.

All programs of the design adopt $\mathrm{C}$ language compiling due to the advantages of $\mathrm{C}$ language in the development of the system. The main program diagram of the system is shown in Fig. 3.

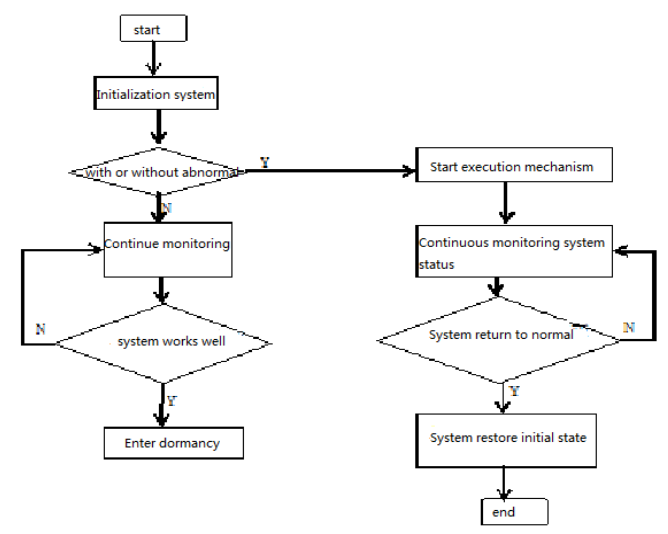

Figure 3. The main flow chart of the system

\section{The Detailed Design of the System}

A/D Switching Circuit Design. The analog signal obtained through the voltage of the sensor received by the single-chip microcomputer will compare with the standard analog signals sent by the zigzag wave of A/D conversion module. That is to say, it is compared though the pin of the single-chip microcomputer with simultaneous development of timer 0 . When the analog signal to be tested is above the standard one, I/O pin signal will change. By this time, the value of timer 0 can become the corresponding digital signals through conversion. The system selects ADC0832 as the A/D conversion chip of the system [6]. 
System Key Design. In order to better control the system, the system is equipped with key control module with six separate keys in connection with P3.0 - P3.5 respectively. According to the definition of programming, the functions are respectively tire pressure upper limit plus, tire pressure upper limit minus, tire pressure lower limit plus, tire pressure lower limit minus, temperature limit plus, temperature limit minus.

The mechanical micro-control key shaking may cause the disorder of the key coding. The key shaking elimination circuit is designed based on this. The specific contents are as follows: The adopted method is to detect the key every other a time period. For instant, the pressed key is confirmed by pressing the key every $5 \mathrm{~ms}$ with continuous scanning of the same key. The confirmation of the scanning times is generally determined by the actual situation with the accumulation time of the scanning time is $50 \mathrm{~ms} \sim 60 \mathrm{~ms}$ at large. For example, if the key is scanned with $5 \mathrm{~ms}$ as the basic time unit, the key can be confirmed by continuous scan of the same key for 11 times to reach $50 \mathrm{~ms}$. It is the same to the detection method of the release of the key. It is determined through repeated detection of the disconnected level of the key status. The advantage of the approach is high efficiency of the program execution with no need to deliberately add delay instruction. Moreover, it is better to determine the key anti-interference ability with the method. The disadvantage is that the program structure is more complicated [7].

Gas Sensor Circuit Design. MPX4105 and MPX4105 types of pressure sensors are adopted by the gas sensor are to design for engine control. This kind of sensor can measure the absolute pressure air of the suction manifold and users can calculate the amount of fuel required for each cylinder according to the measuring results of the absolute pressure of the manifold branch. It integrates the bipolar operational amplifiers, the thin film resistor network and X-type strain gage into one chip to provide high output voltage. The temperature compensation scope is- $40 \sim+125{ }^{\circ} \mathrm{C}$ and the measurement method is the absolute pressure (type A).

Display Circuit Design. The character type liquid crystal display LCD 1602 is selected in the design. It can display two lines with 16 characters each line. Moreover, it uses $+5 \mathrm{~V}$ power supply and it is simple of its peripheral circuit configuration. It is cheap with high cost performance [8].

Temperature Measurement Circuit Design. In order to make it simple of the circuit and the programming, it uses the single bus temperature sensor DS18B20 as temperature sensitive components of the system. There are many advantages of DS18B20 mainly reflecting in less occupation of the hardware resources, simple programming, and high sensitivity and accurate measurement [9].

\section{System Simulation Realization}

There are some differences of Proteus and other simulation software of the single chip microcomputer. It can not only simulate the work situation of the CPU of the single chip microcomputer but also simulate the work situation of the peripheral circuit of the single chip microcomputer or other circuits with no participation of the single chip microcomputer. Therefore, in simulation and program debugging, it is no longer cared about of the change of the contents of registers and storage of the single-chip microcomputer in the execution of certain statements. However, the process and results of program operation and circuit working are directly looked over from the perspective of the engineering. In a certain sense, for such simulation experiments, it is a contradiction and phenomenon to make up the disconnection between the experiment and engineering application.

The tire gauge of the automobile tire gains the analog voltage value corresponding to the automobile tire pressure through the air pressure sensor and process by inputting to the single-chip microcomputer through the V/F transform so as to display the corresponding pressure values in real time. The parameters shall be carefully understood when the digital pressure barometer is designed in case of the damage of the tire gauge due to improper use. The tire gauge of the automobile tire adopts the high-performance absolute pressure sensors with its screen display of the high accurate automobile tire pressure in order to realize the real-time monitoring of the tire pressure. When the automobile tire pressure is in the operation of the abnormal state, it will inform the driver through the alarm. 
Meanwhile, the system also has the function of temperature monitoring to prevent the occurrence of tire burst and achieve the purpose of safe driving[10].

According to the actual situation, there is no ready-made tires and car to test. It simulates under the proteus environment and respectively set up three relays and stepping motors to simulate the tire pressure detection and control process. For the convenience of the test, the design realizes the function by selecting the pressure value directly simulating with the potentiometer. The voltage converts to the pulse with certain frequency through the converter. Then, it converts to the pressure value through pulse counting and show the voltage value through the displayer. The pressure value can be changed through the adjustment of the potentiometer. It will give an alarm when reaching the presupposed threshold value with the yellow light up and the buzzer buzz. The system designs three different kinds of units of measurement and it selects the unit of measurement and set the threshold value through three function selection keys. The overall system circuit under the running condition is shown in Fig. 4.

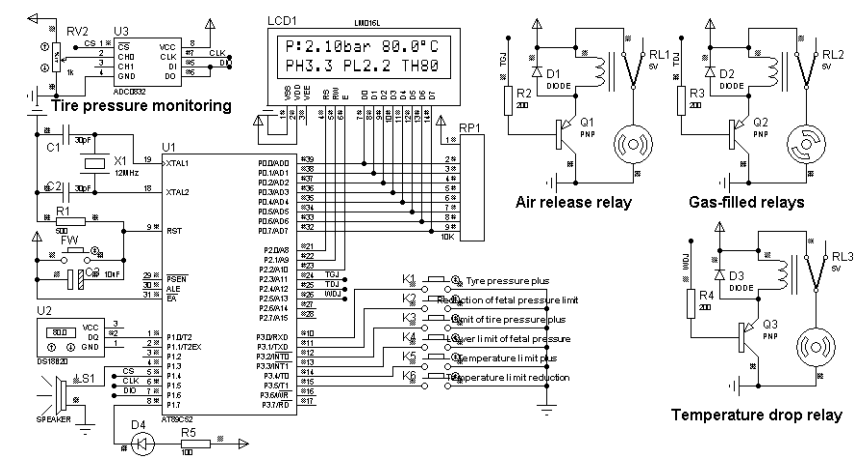

Figure 4. The overall diagram of system operation

\section{Summary}

Through the above introduction, it overall design proves of the system is basically restored. The pulse counts got in the unit time can be gained though the pressure pulse signal received by the single-chip microcomputer. The corresponding actual pressure value is calculated according to the linear relation between the voltage and frequency. In the end, it shows to the users through LCD display circuit. The characteristics of the system are that it can monitor tire pressure condition in real time, and can compensate by invoking the executing agency when the abnormal situations are detected. Meanwhile, it has the tire over temperature alarm function to avoid the tire burst due to excess temperature to a great extent. The system avoids the disadvantages of low precision and simple display of the traditional tire pressure detection system and make the tire pressure detection system designed by the system has the advantages of convenient application, high precision and flexible property, etc under the control of the single-chip microcomputer. Moreover, it can greatly improve the technical indicators of the controlled pressure so as to greatly improve the quality of the products. It can be known that the system is stable in the operation with high precision to reach the expected purpose basically through the simulation under the proteus environment.

\section{References}

[1] Wu Yangquan and Liu Jinxing. Universal Tire Pressure Monitoring System Based on PIC Single-Chip Microcomputer [A] Xihua University Press.

[2] Lei Yini and Lu Yimin. The Design of the Tire Pressure Direct Monitoring System [J] Computer and Digital Engineering, 2005.

[3] Chen Kai, Ni Jiyong and Li Bo. Mining Vehicle Tire Pressure Monitoring Based on Bluetooth [J]. Computer System Application, 2010 (08). 
[4] Zhang Qi, Liu Guofu, Liu Bo and Xie Xiufen. Error Processing and Resonance Frequency Extraction Method [J] Test Technology Journal. 2009(05).

[5] Zhang Jian, Qi Haochen, Yang Wenhua, Hua Zhengfeng and Hu Zhiwen. The Design of a Kind of MEMS Pressure Sensor Used for the Tire Pressure Monitoring [J] Digital Measurement and Instrument Journal. 2015 (10).

[6] He Wenhui. Sensitive Security Guard- Steel General DIY Tire Pressure Monitoring Alarm Apparatus Test [J] Sound Modification Technology. 2012(08).

[7] Liwei and $\mathrm{Xu}$ Wei. On the Tire Intelligent Monitoring System Based on the Single Chip Microcomputer [J] Modern Electronic Technology, 2011.

[8] Song H.J, Hsu H.P, Wiese R. etal. Modeling signal strength range of TPMS in automobiles. Antennas and Propagation Society International Symposium, 2004. IEEE. 2004.

[9] Shen Hongwei. Design Examples and Analysis of Single Chip Microcomputer Application System [M] Beijing: University of Aeronautics and Astronautics Press.

[10]Zhang Xing and Ying Ping. Monitor Design of the Automobile Tire Pressure Monitoring System [J] Electronic Technology, 2005. 